\title{
An Application of ARX Stochastic Models to Iris Recognition
}

\author{
Luis E. Garza Castañón ${ }^{2}$, Saúl Montes de $\mathrm{Oca}^{2}$, and \\ Rubén Morales-Menéndez ${ }^{1}$ \\ ${ }^{1}$ Department of Mechatronics and Automation, ITESM Monterrey Campus, \\ $\{$ legarza, rmm\}@itesm.mx \\ 2 Automation Graduate Program Student, ITESM Monterrey Campus, \\ saul montesdeoca@yahoo.com.mx \\ Av. Eugenio Garza Sada Sur No. 2501 \\ Monterrey, N.L. 64849 México
}

\begin{abstract}
We present a new approach for iris recognition based on stochastic autoregressive models with exogenous input (ARX). Iris recognition is a method to identify persons, based on the analysis of the eye iris. A typical iris recognition system is composed of four phases: image acquisition and preprocessing, iris localization and extraction, iris features characterization, and comparison and matching. The main contribution in this work is given in the step of characterization of iris features by using ARX models. In our work every iris in database is represented by an ARX model learned from data. In the comparison and matching step, data taken from iris sample are substituted into every ARX model and residuals are generated. A decision of accept or reject is taken based on residuals and on a threshold calculated experimentally. We conduct experiments with two different databases. Under certain conditions, we found a rate of successful identifications in the order of $99.7 \%$ for one database and $100 \%$ for the other.
\end{abstract}

\section{Introduction}

Iris recognition is related to the area of biometrics. The main intention of biometrics is to provide reliable automatic recognition of individuals based on the measuring of a physical or behavioral characteristic of persons. Biometrics can be used for access control to restricted areas, such as airports or military installations, access to personal equipments such as laptops and cellular phones, and public applications, such as banking operations [13]. A wide variety of biometrics systems have been deployed and resulting systems include different human features such as: face, fingerprint, hand shape, palmprint, signature, voice and iris [8]. The last one may provide the best solution by offering a much more discriminating power than the others biometrics. Specific characteristics of iris such as a data-rich structure, genetic independence, stability over time and physical protection, makes the use of iris as biometric well recognized.

Please use the following format when citing this chapter:

Castañon, L.E.G., de Oca, S.M., Morales-Menéndez, R., 2006, in IFIP International Federation for Information Processing, Volume 218, Professional Practice in Artificial Intelligence, eds. J. Debenham, (Boston: Springer), pp. 343-352. 
In last years, there have been different implementations of iris recognition systems. Daugman's system [2] used multiscale quadrature wavelets (Gabor filters) to extract texture phase structure information of the iris to generate a 2,048-bit iris code and compared the difference between a pair of iris representations by their Hamming distance. In [11] iris features are extracted by applying a dyadic wavelet transform with null intersections. To characterize the texture of the iris, Boles and Boashash [1] calculated a one dimension wavelet transform at various resolution levels of a concentric circle on an iris image. In this case the iris matching step was based on two dissimilarity functions. Wildes [15] represented the iris texture with a Laplacian pyramid constructed with four different resolution levels and used the normalized correlation to determine whether the input image and the model image are from the same class. A Similar method to Daugman's is reported in [10], but using edge detection approach to localize the iris, and techniques to deal with illumination variations, such as histogram equalization and feature characterization by average absolute deviation. In [7] a new method is presented to remove noise in iris images, such as eyelashes, pupil, eyelids and reflections. The approach is based on the fusion of edge and region information. In [3] an iris recognition approach based on mutual information is developed. In that work, couples of iris samples are geometrically aligned by maximizing their mutual information and subsequently recognized.

In our work we apply standard techniques as integro-differential operators to locate the iris, and histogram equalization over extracted iris area to compensate for illumination variations. The main contribution in this work is given in the step of characterization of iris features by using stochastic ARX models, commonly used by the automatic control community. In our work every iris in database is represented by an ARX model learned from data. In the comparison and matching step, data taken from an arriving iris sample are substituted in every ARX model and residuals are generated. A decision to accept or reject the sample is taken based on the residuals and on a threshold calculated experimentally. The architecture of the proposed method is shown in Fig. 1.

We conduct experiments with UBIRIS database [14] and MILES database [12]. Under certain conditions we found a rate of successful identifications in the order of $99.7 \%$ and $100 \%$ respectively.

\section{THE PROPOSED APPROACH}

The implementation of our approach relies on the use of colored eyes images from UBIRIS and MILES databases. Eyes images include samples where iris is free from any occlusion, and others with moderate obstruction from eyelids and eyelashes. Noisy samples from UBIRIS database are shown in Fig. 2. We transform the images color representation to just grey level pixels, because this process is sufficient to reveal the relevant features of iris.

Our iris recognition system consists of four steps: iris localization and extraction, iris features characterization, and comparison and matching. 


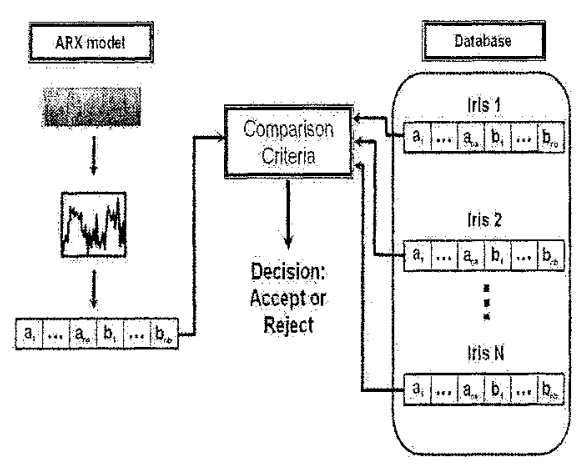

Fig. 1. Architecture of the iris recognition system based on ARX models

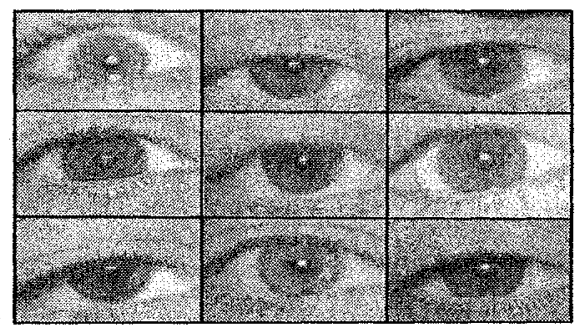

Fig. 2. Eyes samples with noise (moderate obstruction) 


\subsection{Iris Localization}

The search of limbic and pupilar limits is achieved with the use of the integrodifferential operator shown in eqn 1.

$$
\left(r, x_{0}, y_{0}\right)=\left|\frac{\partial}{\partial r} G(r) * \oint_{r, x_{c}, y_{c}} \frac{I(x, y)}{2 \pi r} d s\right|
$$

where $I(x, y)$ is an image containing an eye.

The operator behaves as an iterative circular edge detector, and searches over the image domain $(x, y)$ for the maximum in the partial derivative with respect to an increasing radius $r$, of the normalized contour integral of $I(x, y)$ along a circular arc $d s$ of radius $r$ and center coordinates $\left(x_{0}, y_{0}\right)$. The symbol $*$ denotes convolution and $G_{\sigma}(r)$ is a smoothing function, tipically a Gaussian of scale $\sigma$. The result of this localization operator is shown in Fig. 3.

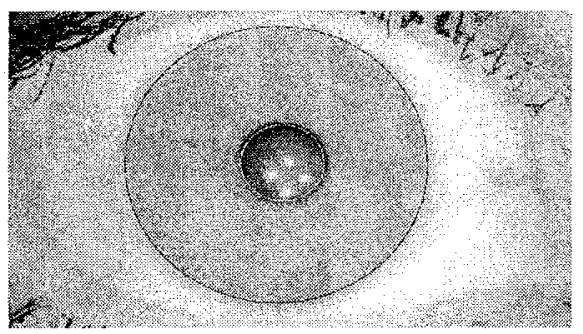

Fig. 3. localization of limbic and pupilar limits with integro-differential operators

This operator behaves well in most cases with moderate noise conditions, but requires some fine tuning of parameters, in order to deal with pupil reflections, obscure eyes and excess of illumination. Heavy occlusion of iris by eyelashes or eyelids needs to be handled by other methods. In our work, eye images with heavy occlusion were discarded.

The extracted iris image has to be normalized to compensate for pupil dilation and contraction under illumination variations. This process is achieved by a transformation from cartesian to polar coordinates, using equations 2 and 3 . The output of this transformation is a rectangular image strip, shown in Fig. 4.

$$
\begin{aligned}
& x(r, \theta)=(1-r) x_{p}(\theta)+r x_{s}(\theta) \\
& y(r, \theta)=(1-r) y_{p}(\theta)+r y_{s}(\theta)
\end{aligned}
$$

where $x(r, \theta)$ and $y(r, \theta)$ are defined as a linear combination of pupil limits $\left(x_{p}(\theta), y_{p}(\theta)\right)$ and limbic limits $\left(x_{s}(\theta), y_{s}(\theta)\right) . r$ is defined in the interval $[0,1]$, and $\theta$ in the interval $[0,2 \pi]$. 


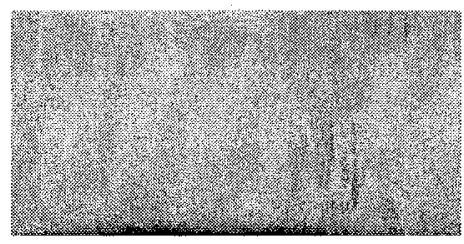

Fig. 4. iris strip image

\subsection{Feature Characterization by ARX Models}

We propose the representation of iris image by an stochastic ARX model. An ARX model represents the behavior of a dynamic system in discrete time [5], where the output $V_{k}$ depends on the input $U_{k}$ and past values of both variables. To represent iris image by an ARX model, we first divide the iris strip image in a rectangular grid, and define output $V_{k}$ as the mean grey level value of every subarea in the grid. The input $U_{k}$ is defined as the corresponding row number of subarea on the grid.

In discrete time the ARX model is defined as follows:

$$
v_{k}=a_{1} v_{k-1}+a_{2} v_{k-2}+\cdots+a_{n a} v_{k-n a}+b_{1} u_{k-1-n d}+\cdots+b_{n b} u_{k-n z b-n d}
$$

Where $a_{1}, a_{2}, \cdots a_{n a}$ and $b_{1} ; b_{2}, \cdots b_{n}$, are the model coefficients to be learned by a least squares (LS) algorithm, $n_{d}$ is an integer number representing the number of times steps that output $V_{k}$ takes to show the effect of a given input $\mathrm{U}_{k}$. This term usually is called dead time. In our case we assume $n_{d}=0$. Former model can be represented as a discrete transfer function in the complex $z$ domain, and is expressed as:

$$
\frac{V(z)}{U(z)}=\frac{b_{1} z^{-1}+b_{2} z^{-2}+\cdots+b_{n b} z^{-n b}}{1-a_{1} z^{-1}-a_{2} z^{-2}-\cdots-a_{n a} z^{-n \alpha}} z^{-n d}
$$

The coefficients $a_{1}, a_{2}, \cdots a_{n a}$ and $b_{1}, b_{2}, \cdots b_{n, b}$ are learned by a least squares (LS) algorithm. This method minimizes an index based on differences between the real data and the model. We define first the following vectors:

$$
\begin{aligned}
\Psi^{T} & =\left[\begin{array}{lllllll}
v_{k-1} & v_{k-2} & \cdots & v_{k-n a} & u_{k-1-n d} & \cdots & u_{k-n b-n d}
\end{array}\right] \\
\theta_{N} & =\left[\begin{array}{lllllll}
a_{1} & a_{2} & \cdots & a_{n a} & b_{1} & \cdots & b_{n b}
\end{array}\right]
\end{aligned}
$$

and then we can rewrite eqn. 4 as follows:

$$
v_{k}=\Psi_{k}^{T} \theta
$$

LS algorithm find the coefficients vector $\theta$ that makes the best estimate of output $v_{k}$, defined as: 


$$
\widehat{v}_{k}=\Psi_{k}^{T} \theta+e_{k}
$$

where $k=n m, n m+a, \ldots, N$ and $n m=\max (n a, n b+n d)$.

difference between real data and model is given by:

$$
e_{N}=V_{N}-\Psi_{N} \theta_{N}
$$

The performance index that LS algorithm minimizes is given by:

$$
J=\sum_{k=n ? n}^{N} e_{k}^{2}=e_{N}^{T} e_{N}
$$

Iris database then is composed by an ARX model for every iris. When an iris arrives for recognition, the comparison is made by using eqn. (11), and obtaining the error in eqn. (10), where $V_{N}$ and $\theta_{N}$ belong to iris in database $\left(I^{T}\right)$ and matrix $\Psi_{N}$ belongs to iris simple $\left(I^{S}\right)$, this lead us to following equations:

$$
e_{N}=V_{N}^{I^{T}}-\Psi_{N}^{I^{S}} \theta_{N}^{I^{T}}
$$

where $e_{N}, V_{N}^{I^{T}}, \Psi_{N}^{I^{S}}$ y $\theta_{N}^{I^{T}}$ are defined as:

$$
\begin{aligned}
& e_{N}=\left[\begin{array}{c}
e_{n m} \\
e_{n m+1} \\
\vdots \\
e_{N}
\end{array}\right], V_{N}^{I^{T}}=\left[\begin{array}{c}
v_{n m}^{I^{T}} \\
v_{n m+1}^{I^{T}} \\
\vdots \\
v_{N}^{I^{T}}
\end{array}\right] \\
& \Psi_{N}^{I^{S}}=\left[\begin{array}{cccccc}
v_{n m-1}^{I^{S}} & \cdots & v_{n m-n a}^{I^{S}} & u_{n m-1-n d}^{I^{S}} & \cdots & u_{n m-n b-n d}^{I^{S}} \\
v_{n m}^{I^{S}} & \cdots & v_{n m-n a+1}^{I^{S}} & u_{n m-n d}^{I^{S}} & \cdots & u_{n m-n b-n d+1}^{I^{S}} \\
\vdots & \ddots & \vdots & \vdots & \ddots & \vdots \\
v_{N-1}^{I^{S}} & \cdots & v_{N-n a}^{I^{S}} & u_{N-1-n d}^{I^{S}} & \cdots & u_{N-n b-n d}^{I^{T}}
\end{array}\right], \theta_{N}^{I^{T}}=\left[\begin{array}{c}
a_{1}^{I^{T}} \\
a_{2}^{I^{T}} \\
\vdots \\
a_{n a}^{I^{T}} \\
b_{1}^{I^{T}} \\
\vdots \\
b_{n b}^{I^{T}}
\end{array}\right]
\end{aligned}
$$

\section{$3 \quad$ Experiments}

Experiments were ran for UBIRIS and MILES databases. Images with too much occlusion and noise were discarded, because the difficulty to locate the iris region with integro-differential operators. Then, our UBIRIS experimental database was built with 1013 samples coming from 173 users, and MILES database consists of 


\begin{tabular}{|c|c|c|c|}
\hline$D B$ Size (\%) & \# of iris & \# Threshold samples & \# Test samples \\
\hline 100 & 1013 & 52 & 788 \\
\hline 90 & 912 & 46 & 693 \\
\hline 80 & 811 & 42 & 596 \\
\hline 70 & 710 & 36 & 501 \\
\hline 50 & 507 & 26 & 308 \\
\hline
\end{tabular}

Table 1. UBIRIS Databases used for experiments with 173 users. First column refers to the percent of database used. For instance, $90 \%$ means that $10 \%$ of worst user samples were discarded. Second column refers to total number of iris samples, third column refers to the number of samples used to calculate the decision threshold, and fourth column refers to the total number of samples used for testing.

\begin{tabular}{|c|c|c|c|}
\hline$D B$ Size (\%) & \# of iris & \# Threshold samples & \# Test samples \\
\hline 100 & 36 & 10 & 167 \\
\hline 75 & 36 & 8 & 116 \\
\hline
\end{tabular}

Table 2. MILES Databases used for experiments with 119 users.

\begin{tabular}{|c|c|c|c|c|c|c|}
\hline grid size & $\begin{array}{c}100 \% \\
\text { DB }\end{array}$ & $\begin{array}{c}90 \% \\
\text { DB }\end{array}$ & $\begin{array}{c}\mathbf{8 0 \%} \\
\text { DB }\end{array}$ & $\begin{array}{c}\mathbf{7 0 \%} \\
\text { DB }\end{array}$ & $\begin{array}{c}\mathbf{6 0 \%} \\
\text { DB }\end{array}$ & $\begin{array}{c}\mathbf{5 0 \%} \\
\text { DB }\end{array}$ \\
\hline $\mathbf{4} \times \mathbf{4}$ & 84.64 & 85.39 & 93.42 & 96.65 & 97.93 & 99.7 \\
\hline $\mathbf{5} \times \mathbf{5}$ & 86.31 & 87.82 & 91.69 & 94.23 & 97.93 & 98.8 \\
\hline $\mathbf{8} \times \mathbf{8}$ & 83.21 & 84.98 & 86.68 & 92.36 & 94.25 & 95.51 \\
\hline $\mathbf{1 0} \times \mathbf{1 0}$ & 80.12 & 80.38 & 83.54 & 88.64 & 86.67 & 90.12 \\
\hline
\end{tabular}

Table 3. Results of experiments for UBIRIS database (in \% of accuracy) with different grid size. Best results are highlighted

\begin{tabular}{|c|c|c|}
\hline grid size & $\begin{array}{c}100 \% \\
\text { DB }\end{array}$ & $\begin{array}{c}\mathbf{7 5} \% \\
\text { DB }\end{array}$ \\
\hline $\mathbf{4 \times 4}$ & 91.53 & 1000 \\
\hline $\mathbf{5} \times \mathbf{5}$ & 01.35 & 100.0 \\
\hline $\mathbf{8} \times \mathbf{8}$ & 93.41 & 99.19 \\
\hline $\mathbf{1 0} \times \mathbf{1 0}$ & 93.79 & 99.19 \\
\hline
\end{tabular}

Table 4. Results of experiments for MILES database (in \% of accuracy) with different grid size. Best results are highlighted 
213 samples from 199 users. With these databases, we perform some experiments with $100 \%$ of samples and others experiments where worst user samples were discarded. Tables 1 and 2 shows the different databases used.

The order of ARX model (number of coeffficients $a_{i}, b_{i}$ ) was determined empirically by doing differents experiments, and best results were obtained for $n_{a}=5$ and $n_{b}=5$ in both, UBIRIS and MLLES databases. In tables 3 and 4 these results are shown. We can see that best results were obtained for cleaner databases.

In Fig. 5 we can see the ROC curves for UBIRIS databases used in experiments. Databases with cleaner iris samples reflects better results. In Fig. 6 we can see the authentic-impostor distribution curves for two UBIRIS databases used. The Overlapping between distribution curves in Fig. 6 (a) leads to worst results.

Similar ROC and authentic-impostor curves were obtained for MILES database experiments and are not shown.

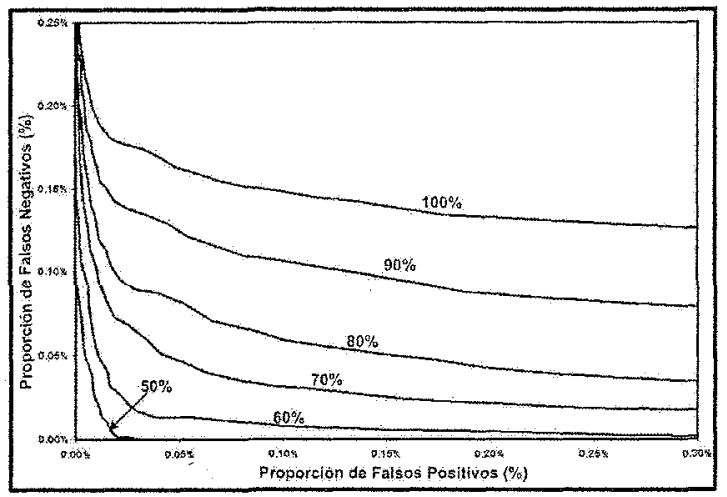

Fig. 5. Receiver operating characteristic (ROC) curve for experiments with different databases.

\section{Comparison to Previous Work}

Daugman's system [2] has been tested thoroughly with databases containing thousands of samples, and reports of $100 \%$ of accuracy have been given. In [11], the experimental results given are in the order of $97.9 \%$, by working with a database of 100 samples from 10 persons. Boles and Boashash [1] report best results in the order of $100 \%$ but working with very small sets of images. Wildes [15] report results in the order of $100 \%$ by working with a database of 600 samples coming from 40 individuals. In [10], a report is given about a performance of $99.09 \%$ in experiments with a database of 500 iris images from 25 individuals. In [7] the results are between $98 \%$ and $99 \%$ by working with CASIA database 


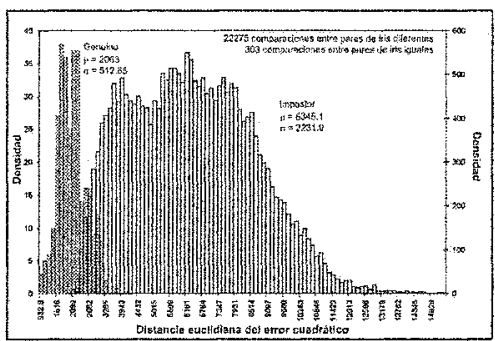

(a)

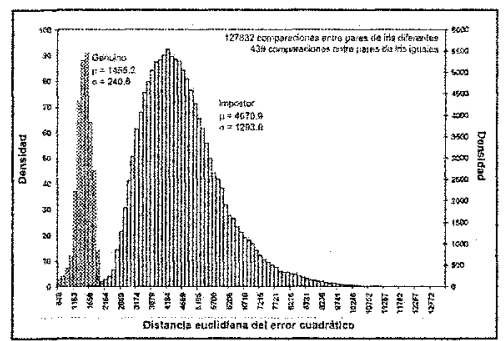

(b)

Fig. 6. authentic-impostor distribution for ARX based system. (a) database with $100 \%$ of data. (b) database with $50 \%$ of data.

(2255 samples from 213 subjects). In [3] best results are in the order of $99.05 \%$ with a database of 384 images from 64 persons.

None of these works specify the quality of databases, so a direct comparison is not possible. What we can say is that we are competitive with most methods when our method work with clean databases, which means eye images with no obstruction and noise. Our best results were $99.7 \%$ for UBIRIS database and $100 \%$ for MILES database obtained with cleanest databases.

\section{Conclusions and Future Works}

A new approach for iris recognition has been presented. The novel contribution relies on the feature characterization of iris by the use of stochastic ARX models. Although experimental results show better results for databases with cleaner eyes images, we are looking forward to improve the methodology by combining statistical sampling methods and stochastic models. We believe the combination of best aspects of both approaches will lead us to a more robust and accurate iris identification system.

\section{References}

1. W. Boles and B. Boashash, "Iris Recognition for Biometric Identification using dyadic wavelet transform zero-crossing", IEEE Transactions on Signal Processing, Vol, 46, No. 4, 1998, pp. 1185-1188.

2. J. Daugman, "How Iris Recognition Works", IEEE Transactions on Circuits and Systems for Video Technology, Vol. 14, No. 1, 2004, pp. 21-30.

3. M. Dobes, L. Machala, P. Tichasvky, and J. Pospisil, "Human Eye Iris Recognition Using The Mutual Information", Optik, No. 9, 2004, pp. 399-404.

4. A. Efros, and T. Leung, "Texture Synthesis by Non-Parametric Sampling", in Proceedings of the 7th IEEE International Conference on Computer Vision, September 1999, Vol. 2, pp. 1033-1038. 
5. G. Franklin, J. Powell, and M. Workman, Digital Control of Dynamic Systems, Addison-Wesley, 3a edition, 1997

6. J. Harnmersley, "Monte Carlo Methods for Solving Multivariate Problems", Annals of New York Academy of Science, 1960, No. 86, pp. 844-874.

7. J. Huang, Y. Wang, T. Tan, and J. Cui, "A New Iris Segmentation Method for Iris Recognition System", In Proceedings of the 17th International Conference on Pattern Recognition, 2004, pp. 554-557.

8. A. Jain, A. Ross, A. Prabhakar, "An Introduction to Biometric Recognition", IEEE Transactions on Circuits and Systems for Video Technology, Vol. 14, No. 1, 2004, pp. $4-20$.

9. L. Liang; C. Liu, Y. Xu, B. Guo, and H. Shum, "Real-time Texture Synthesis by Patch-based Sampling", ACM Transactions on Graphics, Vol. 20, No. 3, July 2001, pp. $127-150$.

10. L. Ma, Y. Wang, T. Tan, and D. Zhang, "Personal Identification Based on Iris Texture Analysis", IEEE Transactions on Pattern Analysis and Machine Intelligence, Vol. 25, No. 12, 2003, pp. 1519 - 1533.

11. D. de Martin-Roche, C. Sanchez-Avila, and R. Sanchez-Reillo, "Iris Recognition for Biometric Identification using dyadic wavelet transform zero-crossing", In Proceedings of the IEEE 35th International Conference on Security Technology, 2001, pp. 272-277.

12. Miles Research. Sample iris Pictures. http://www.milesresearch.com/

13. M. Negin, Chmielewski T., Salganicoff M., Camus T., Cahn U., Venetianer P., and Zhang G. "An Iris Biometric System for Public and Personal Use ", Computer, Vol. 33 , No. 2,2000 , pp. $70-75$.

14. H. Proenca, and L. Alexandre,"UBIRIS: A Noisy Iris Image Database", in Proceedings of the International Conference on Image Analysis and Processing 2005, Vol. 1 , pp. $970-977$.

15. R. Wildes, "Iris Recognition: An Emerging Biometric Technology", Proceedings of the IEEE, Vol. 85 , No. 9, 1997, pp. 1348-1363. 